\title{
Thermodynamics and area in Minkowski space: Heat capacity of entanglement.
}

\author{
Ram Brustein, Amos Yarom \\ Department of Physics, Ben-Gurion University, Beer-Sheva 84105, Israel \\ E-mail: ramyb@bgumail.bgu.ac.il, yarom@bgumail.bgu.ac.il
}

\begin{abstract}
Tracing over the degrees of freedom inside (or outside) a sub-volume $V$ of Minkowski space in a given quantum state $|\psi\rangle$, results in a statistical ensemble described by a density matrix $\rho$. This enables one to relate quantum fluctuations in $V$ when in the state $|\psi\rangle$, to statistical fluctuations in the ensemble described by $\rho$. These fluctuations scale linearly with the surface area of $V$. If $V$ is half of space, then $\rho$ is the density matrix of a canonical ensemble in Rindler space. This enables us to 'derive' area scaling of thermodynamic quantities in Rindler space from area scaling of quantum fluctuations in half of Minkowski space. When considering shapes other than half of Minkowski space, even though area scaling persists, $\rho$ does not have an interpretation as a density matrix of a canonical ensemble in a curved, or geometrically non-trivial, background.
\end{abstract}

\section{INTRODUCTION}

The microscopic origin of Black hole (BH) thermodynamics, and in particular area scaling of black-hole entropy and other thermodynamical quantities is still not well understood even many years after its discovery. There have been many attempts to explain BH thermodynamics. One of the ideas underlying several proposals is that the horizon prevents observers from having access to the whole set of quantum states, and hence the entropy they measure is due to entanglement.

In an attempt to understand BH entropy Srednicki [1], (and previously Bombelli et. al. 2]) considered the von Neumann entropy of quantum fields in a state described by the density matrix $\rho_{i n}$, defined by taking the vacuum state and tracing over the degrees of freedom (DOF) external to a spherical sub-volume of Minkowski space. Thus, effectively reducing the DOF available to an observer, to the inside of the spherical sub-volume. They 
discovered that this "entropy of entanglement" was proportional to the surface area of the sphere. Using general arguments it is possible to show that the entropy obtained by tracing over the DOF outside of a sub-volume of any shape is equal to the one obtained by tracing over the DOF inside this sub-volume. However, a numerical calculation was needed in order to show that it is linearly dependent on the area (for a spherical sub-volume). Following this line of thought, some other geometries were considered, in particular, half of Minkowski space in various dimensionalities (see for example, $[3,44,5], 6$, 7]).

We offer a novel point of view and a new calculational tool to establish area scaling properties of statistical averages in such a setup. Recently, we have shown [8] that the variance of a class of operators restricted to the interior/exterior of a sub-volume $V$ of Minkowski space scale linearly with the boundary area of the sub-volume. Specifically, we have considered operators, $O$, in $\mathrm{d}+1$ dimensional Minkowski space, which are obtained by integrating over an operator density $\mathcal{O}: O=\int \mathcal{O} d^{d} x$. One can restrict such operators to the inside of a sub-volume $V$ by defining $O^{V}=\int_{V} \mathcal{O} d^{d} x$. Under certain conditions, fluctuations of $O^{V}$ in the vacuum state $\left\langle 0\left|\left(O^{V}-\left\langle O^{V}\right\rangle\right)^{2}\right| 0\right\rangle$, scale linearly with the boundary area of $V$. In this setup, the vacuum state, being a superposition of external products of states in the 'in' and 'out' region, is an entangled state.

To show how area scaling of fluctuations of operators in the vacuum of Minkowski space are related to area dependence of thermodynamic quantities, we recall that expectation values of operators restricted to a certain region of space may be described by an average over a mixed state described by a density matrix $\rho_{i n}$ [9] (see eq. (II)). One may then derive area scaling of statistical averages in the system described by $\rho_{i n}$, from area scaling properties of quantum fluctuations in the Minkowski vacuum [8]. Since fluctuations of operators are related to thermodynamic quantities, we can establish that the thermodynamics described by $\rho_{\text {in }}$ scales linearly with the surface area of $V$. Equation (11) and the properties of $\rho_{i n}$ are reviewed in section [I]

As an explicit example of this relation, we consider the case where the volume in question is half of space. This was studied earlier by [3, 6, 10], where it was shown that $\rho_{i n}=\left\langle e^{-\beta_{0} H_{R}}\right\rangle$, $H_{R}$ and $\beta_{0}$ being the Rindler Hamiltonian and inverse temperature. Equation (1) allows us to relate the energy fluctuations in Rindler space to fluctuations of the Rindler Hamiltonian, in the Minkowski vacuum. As is well known, energy fluctuations are a measure of the heat capacity. Therefore, we have here another method by which one can show that the heat 
capacity of Rindler space scales linearly with the surface area of the horizon. This is done in section

Obviously, one would like to know if tracing over DOF inside (or outside) sub-volumes of general shape would correspond to other spaces, with properties that are, perhaps, similar to Rindler space. That is, we may always define an effective Hamiltonian $\left\langle e^{-\beta_{0} H_{e f f}}\right\rangle=\rho_{i n}$, and ask if $H_{\text {eff }}$ describes a system in a static curved background. We show that for shapes other than half of space, it is not. This is done in section IV We conclude with a summary and discussion of our results.

\section{PROPERTIES OF THE DENSITY MATRIX $\rho_{i n}$.}

The density matrix describing the Minkowski vacuum state is $\rho=|0\rangle\langle 0|$. Now define $\rho_{\text {in }}=$ Trace $_{\text {out }} \rho$. Where 'Trace out $^{\prime}$ means tracing over those states which are external to the sub-volume $V$. The density matrix obtained this way has many interesting properties $[1,3,4,6,69,10]$. We review some of them.

First, we wish to recall the correspondence between quantum expectation values of operators $O^{V}$ restricted to a sub-volume $V$, and statistical averages in a system described by a density matrix $\rho_{i n}$. This is a well known feature of quantum mechanics, and it is essential for our discussion. Writing $|0\rangle=\sum A_{\alpha, a}|a\rangle \otimes|\alpha\rangle$, where $|a\rangle$ is a state inside the volume $V$ and $|\alpha\rangle$ is external to it, it follows that $\rho_{\text {in }}=\sum_{\gamma}\langle\gamma \mid 0\rangle\langle 0 \mid \gamma\rangle=\sum A_{\alpha, a}|a\rangle\langle b| A_{b, \alpha}^{\dagger}$. One may now show, by expressing $O^{V}$ in the 'in' basis, and performing some straightforward algebra [9], that

$$
\left\langle 0\left|O^{V}\right| 0\right\rangle=\operatorname{Tr}\left(\rho_{i n} O^{V}\right),
$$

so that expectation values of such operators in the vacuum of Minkowski space are equal to expectation values of operators in the system described by the density matrix $\rho_{i n}$.

Next, we wish to find an effective Hamiltonian $H_{\text {eff }}$ which is proportional to the logarithm of $\rho_{i n}$. This can be done using an extension of the method introduced in [10]: we shall write out both $\rho_{i n}$ and $\left\langle e^{-\beta_{0} H_{e f f}}\right\rangle$ as a path integral, and compare the expressions.

Switching to a Euclidian metric, it is possible to represent the wave functional of the vacuum as $\langle 0 \mid \psi(\vec{x})\rangle=\int_{\varphi(\vec{x}, 0)=\psi(\vec{x})} \exp \left[-\int_{0}^{\infty} \ldots \int \mathcal{L} d^{d} x d \tau\right] D \varphi$, where the integral is over all fields evaluated at $t>0$, which satisfy the boundary conditions $\varphi(\vec{x}, 0)=\psi(\vec{x})$. In the 
specific case of interest,

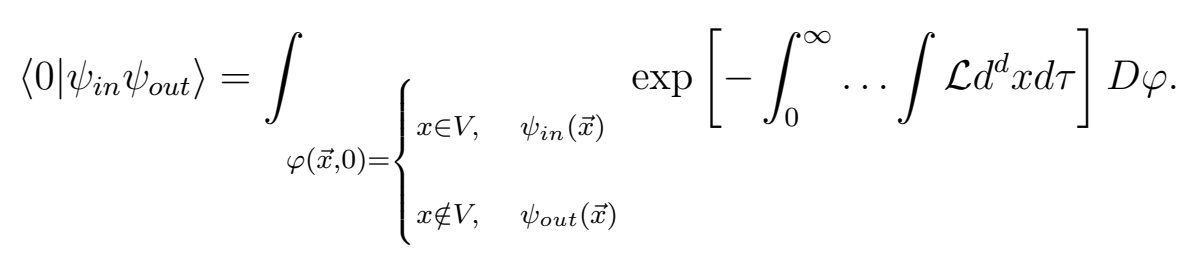

It follows that

$$
\left\langle\psi_{i n}^{\prime}\left|\rho_{i n}\right| \psi_{i n}^{\prime \prime}\right\rangle=\int_{\varphi(\vec{x}, 0)= \begin{cases}x \in V, t=0^{+}, & \psi_{i n}^{\prime}(\vec{x}) \\ x \in V, t=0^{-}, & \psi_{i n}^{\prime \prime}(\vec{x})\end{cases} } \exp \left[-\int_{-\infty}^{\infty} \cdots \int \mathcal{L} d^{d} x d \tau\right] D \varphi .
$$

The effective Hamiltonian $H_{\text {eff }}$, is found by comparing expression (2) to that for matrix elements of $e^{-\beta_{0} H_{e f f}}[4,[5]$. To obtain these, we observe that

$$
\begin{aligned}
& \left\langle\psi_{i n}^{\prime}\left|e^{-\beta_{0} H_{e f f}}\right| \psi_{i n}^{\prime \prime}\right\rangle \\
& =\int_{\substack{\varphi(x, 0)=\psi_{i n}^{\prime}(\vec{x}) \\
\varphi\left(x,-i \beta_{0}\right)=\psi_{i n}^{\prime \prime}(\vec{x})}} \exp \left[\imath \int_{0}^{\imath \beta_{0}}\left(\int \pi \frac{d \varphi}{d t} d^{d} x-H_{e f f}\right) d t\right] D \varphi D \pi .
\end{aligned}
$$

This equality is obtained by time slicing the interval $\left[0, \beta_{0}\right]$ into infinitesimal values, and using the fact that $e^{-\beta_{0} H_{\text {eff }}}$ is the generator of time translations for imaginary time, from $t=0$ to $t=-\imath \beta_{0}$. One must assume here that $H_{\text {eff }}$ is time independent. Note that $\beta_{0} \neq 0$.

Changing the time variable to $\tau=i t,\left\langle e^{-\beta_{0} H_{e f f}}\right\rangle$ reduces to:

$$
\left\langle e^{-\beta_{0} H_{e f f}}\right\rangle=\int_{\substack{\varphi(x, 0)=\psi_{i n}^{\prime}(\vec{x}) \\ \varphi\left(x, \beta_{0}\right)=\psi_{i n}^{\prime \prime}(\vec{x})}} \exp \left[\int_{0}^{\beta_{0}}\left(\int \imath \pi \dot{\varphi} d^{d} x-H_{e f f}\right)(d \tau)\right] D \varphi D \pi .
$$

If we are to compare equation (3) with equation (2) we must make some sort of guess about the nature of $H_{\text {eff }}$. We therefore assume that $H_{\text {eff }}$ is the Hamiltonian of a free field theory in a static, curved background. Since $H_{\text {eff }}$ is the generator of time translations, it is also the Legendre transform of a Lagrangian. A free field Lagrangian density in a gravitational background with metric $g_{\mu \nu}$ is given by $\mathcal{L}=-\frac{1}{2} \sqrt{-g} g^{\mu \nu} \partial_{\mu} \varphi \partial_{\nu} \varphi$. In order to emphasize that this is not necessarily a Euclidian parametrization, we write the space-time coordinates as $(\vec{\xi}, \eta)$. Considering static coordinates, for which $g^{0 i}=0$, and $\frac{d}{d x^{0}} g^{\mu \nu}=0$, then gives us $H_{\text {eff }}=\int \mathcal{H} \sqrt{h} d^{d} x$, where $\mathcal{H}=\sqrt{-g_{00}}\left(\frac{1}{2}\left(\frac{\pi}{\sqrt{h}}\right)^{2}+\frac{1}{2} h^{i j} \partial_{i} \varphi \partial_{j} \varphi\right), h^{i j}$ are the space-space components of the metric $\left(h^{i j}=g^{i j}\right)$, and $\pi=\frac{\dot{\varphi} \sqrt{h}}{\sqrt{-g_{00}}}$. 
Performing the Gaussian integral and using $\Omega=\frac{1}{\sqrt{g_{00}^{E}}}$, (where we have used $g_{00}^{E}=-g_{00}$ ), one obtains the following expression [4],

$$
\left\langle\psi_{i n}^{\prime}\left|e^{-\beta_{0} H_{e f f}}\right| \psi_{i n}^{\prime \prime}\right\rangle=\int_{\substack{\varphi(\xi, 0)=\psi_{i n}^{\prime}(\xi) \\ \varphi\left(\xi, \beta_{0}\right)=\psi_{i n}^{\prime \prime}(\xi)}} \exp \left[-\int_{0}^{\beta_{0}} \int \mathcal{L}_{E} d^{d} \xi d \eta\right]|\Omega||g|^{\frac{1}{4}} D \varphi .
$$

If we find a metric under which equations (21), and (4) are equal, then $H_{\text {eff }}$ would be the Hamiltonian of a free field theory in a static background given by $g^{\mu \nu}$. In order for such an equality to be satisfied we need: (1) That the boundary conditions be identical. (2) That the actions be identical. (3) That the measures be identical. In order to satisfy the three conditions, we wish to find a coordinate transformation from a Minkowski $(\vec{x}, t)$ system, to a $(\vec{\xi}, \eta)$ system. In this coordinate system we immediately get:

1. The boundary conditions on the path integral are met if

$$
\begin{aligned}
& \left\{\left(\vec{x}, 0^{+}\right) \mid \vec{x} \in V\right\}=\left\{(\vec{\xi}, 0) \mid \vec{\xi} \in I_{\xi}\right\} \\
& \left\{\left(\vec{x}, 0^{-}\right) \mid \vec{x} \in V\right\}=\left\{\left(\vec{\xi}, \beta_{0}\right) \mid \vec{\xi} \in I_{\xi}\right\} .
\end{aligned}
$$

2. The actions will be equal only if (a) $\mathcal{L}$ is the Lagrangian density of a free field theory in a Minkowski space background. So $(\vec{\xi}, \eta)$ constitute a coordinate transformation of Minkowski space. (b) The limits of integration on the Lagrangian densities are equal. This requires that the transformation $t \rightarrow t(\vec{\xi}, \eta), \vec{x} \rightarrow(\vec{\xi}, \eta)$, be onto, such that the range of $\eta$ is $\left[0, \beta_{0}\right]$.

3. There is an incompatibility of the measure [4, 5], which we may ignore, since for our purposes it is a field independent multiplicative factor in the path integral.

If $V$ is half of space, then as explicitly shown in 10$]$, the $(\vec{\xi}, \eta)$ coordinate system is a polar coordinate system in the (x-t) plane of Minkowski space. Such a coordinate system describes Rindler space. The effective Hamiltonian, $H_{e f f}$, is the Rindler Hamiltonian $H_{R}$, and $\beta_{0}=\frac{2 \pi}{a}$, where $a$ is a dimensional constant introduced so that $\eta$ will have units of length. Combining this observation with equation (10), we see that expectation values of fluctuations of operators in half of Minkowski space are equal to expectation values of the same operators in Rindler space at some temperature $\beta_{0}$,

$$
\left\langle\left(\Delta O^{V_{1 / 2}}\right)^{2}\right\rangle=\operatorname{Trace}\left(\rho_{i n}\left(\Delta O^{V_{1 / 2}}\right)^{2}\right)=\operatorname{Trace}\left(e^{-\beta_{0} H_{R}}\left(\Delta O^{V_{1 / 2}}\right)^{2}\right) .
$$


Looking at eq.(6) from right to left, then, since Rindler space thermodynamics is known to be area-dependent [4, 5, 11], we should not be surprised that fluctuations of these operators scale linearly with the surface area of the horizon. An alternate viewpoint is that we have "derived" area-thermodynamcs of Rindler space from "known" area scaling of vacuum expectation values in Minkowski space.

\section{THE HEAT CAPACITY OF RINDLER SPACE RADIATION.}

As a concrete example of the usage of equation (6), we calculate the heat capacity of Rindler space radiation. Generally, the heat capacity is proportional to energy fluctuations: $\left\langle(\Delta E)^{2}\right\rangle=C_{V} T^{2}$. Therefore, the area dependence of the heat capacity of Rindler space is given by the vacuum expectation value of fluctuations of the Rindler Hamiltonian (equation (11) ),

$$
C_{V} T^{2}=\operatorname{Trace}\left(e^{-\beta_{0} H_{R}}\left(\Delta H_{R}\right)^{2}\right)=\left\langle\left(\Delta H_{R}\right)^{2}\right\rangle
$$

This, in turn, has area scaling properties due to the general argument given in [8, 12], and as shown explicitly below.

We shall use the $d+1$ dimensional Rindler metric in the form:

$$
d s^{2}=-e^{2 a \xi} d \eta^{2}+e^{2 a \xi} d \xi^{2}+d \vec{x}_{\perp}^{2}
$$

that is obtained from the Minkowski space metric:

$$
d s^{2}=-d t^{2}+d z^{2}+d \vec{x}_{\perp}^{2}
$$

by a coordinate transformation:

$$
\begin{aligned}
t(\xi, \eta) & =\frac{1}{a} e^{a \xi} \sinh a \eta \\
z(\xi, \eta) & =\frac{1}{a} e^{a \xi} \cosh a \eta \\
\vec{x}_{\perp} & =\vec{x}_{\perp} .
\end{aligned}
$$

Here $\vec{x}_{\perp}$ stands for the $d-1$ transverse coordinates.

To find the energy fluctuations of Rindler space, we first find an expression for the Rindler 
space Hamiltonian:

$$
\begin{aligned}
H_{R} & =\int \sqrt{h} \sqrt{-g_{00}}\left[\frac{1}{2}\left(\frac{\pi}{\sqrt{h}}\right)^{2}+\frac{1}{2} h^{i j} \partial_{i} \varphi \partial_{j} \varphi\right] d \xi d \vec{x}_{\perp} \\
& =\int e^{a \xi} e^{a \xi}\left[\frac{1}{2}\left(\partial_{\eta} \varphi\right)^{2} e^{-2 a \xi}+\frac{1}{2}\left(\left(\partial_{\xi} \varphi\right)^{2} e^{-2 a \xi}+\left(\partial_{\vec{x}_{\perp}} \varphi\right)^{2}\right)\right] d \xi d \vec{x}_{\perp} \\
& =\frac{1}{2} \int\left[\left(\partial_{\eta} \varphi\right)^{2}+\left(\partial_{\xi} \varphi\right)^{2}+e^{2 a \xi}\left(\partial_{\vec{x}_{\perp}} \varphi\right)^{2}\right] d \xi d \vec{x}_{\perp} .
\end{aligned}
$$

To get acquainted with this operator we compare it to the Minkowski space energy operator in half space, given by:

$$
E_{M+}=\frac{1}{2} \int_{z>0}\left[\left(\partial_{t} \varphi\right)^{2}+\left(\partial_{z} \varphi\right)^{2}+\left(\partial_{\vec{x}_{\perp}} \varphi\right)^{2}\right] d z d \vec{x}_{\perp},
$$

where $z$ is the coordinate along which space is divided into two halves $(z>0$ and $z<0)$. In order to relate $H_{R}$ and $E_{M+}$ we note that:

$$
\left(\begin{array}{c}
\partial_{t} \\
\partial_{z}
\end{array}\right)=e^{-a \xi}\left(\begin{array}{cc}
\cosh a \eta & -\sinh a \eta \\
-\sinh a \eta & \cosh a \eta
\end{array}\right)\left(\begin{array}{l}
\partial_{\eta} \\
\partial_{\xi}
\end{array}\right),
$$

therefore, evaluating $E_{M+}$ at an equal time contour, we have

$$
E_{M+}=\frac{1}{2} \int e^{-a \xi}\left[\left(\partial_{\eta} \varphi\right)^{2}+\left(\partial_{\xi} \varphi\right)^{2}+e^{2 a \xi}\left(\partial_{\vec{x}_{\perp}}\right)^{2}\right] d \xi d \vec{x}_{\perp}
$$

On the other hand, evaluating $H_{R}$ at $\eta=0$, we have

$$
\begin{aligned}
H_{R} & =\frac{1}{2} \int e^{a \xi}\left[(\pi)^{2}+\left(\partial_{z} \varphi\right)^{2}+\left(\partial_{\vec{x}_{\perp}}\right)^{2}\right] d z d \vec{x}_{\perp} . \\
& =\frac{1}{2} \int(a z)\left[(\pi)^{2}+\left(\partial_{z} \varphi\right)^{2}+\left(\partial_{\vec{x}_{\perp}}\right)^{2}\right] d z d \vec{x}_{\perp} .
\end{aligned}
$$

From equation (91), we see that the energy density in half of Minkowski space is equal to the Rindler energy density restricted to a region close to the Rindler horizon $(\xi \rightarrow-\infty)$.

Now that we have an expression for the Hamiltonian of Rindler space in terms of the coordinates of Minkowski space, we may evaluate the energy fluctuations in Rindler space by using equation (11),

$$
\left\langle 0_{M}\left|\left(: H_{R}:\right)^{2}\right| 0_{M}\right\rangle=\text { Trace }\left[e^{-\beta_{0} H_{R}}\left(: H_{R}:\right)^{2}\right]
$$

We carry out this calculation in the appendix. The result is

$$
\left\langle\left(: H_{R}:\right)^{2}\right\rangle=V_{\perp} a^{2} \Lambda^{d-1} \frac{(d+1) \Gamma^{2}\left(\frac{d+1}{2}\right)}{(d-1) 2^{d+5} \pi^{1+\frac{d}{2}} \Gamma\left(2+\frac{d}{2}\right)},
$$


where $\Lambda$ is the UV cutoff of the theory, $V_{\perp}$ is the area of the boundary between the two halves of Minkowski space and $\frac{a}{2 \pi}$ is the Rindler temperature. The heat capacity is then given by:

$$
C=\frac{\left\langle\Delta E^{2}\right\rangle}{T^{2}}=\frac{V_{\perp}}{\Lambda^{2}} \frac{(d+1) \Gamma^{2}\left(\frac{d+1}{2}\right)}{(d-1)^{2} 2^{d+5} \pi^{1+\frac{d}{2}} \Gamma\left(2+\frac{d}{2}\right)},
$$

which scales linearly with the surface area of half space.

We summarize what we have learned so far: area scaling is a general property of fluctuations of a certain class of operators restricted to a sub-volume of Minkowski space. Area dependence of the heat capacity of Rindler space radiation, is a special case of this general property, when the volume is chosen to be half of Minkowski space.

\section{IV. $H_{e f f}$ FOR OTHER SHAPES.}

Equation (11) suggests that the area scaling of the entangled system in Minkowski space 12] and the thermodynamics of the space given by the Hamiltonian $H_{\text {eff }}$ are two aspects of the same phenomenon. As shown explicitly in the previous section, this allows one to obtain, for example, area scaling of the heat capacity of a free field in Rindler space from area scaling of quantum fluctuations in half of Minkowski space.

One would wish to show a similar equivalence for sub-volumes of other shapes. However, as we will show, for volumes other than half space, the effective Hamiltonian obtained cannot be that of a non interacting field theory in a static curved, or geometrically non-trivial, background.

Let us consider a general sub-volume $V$. Equating equations (2) and (41), we wish to find an onto coordinate transformation

$$
\begin{aligned}
& t=t(\eta, \vec{\xi}) \\
& \vec{x}=\vec{x}(\eta, \vec{\xi}),
\end{aligned}
$$

such that $\eta \in\left[0, \beta_{0}\right]$, and $\vec{\xi} \in I_{\xi}$, and $\frac{d}{d t} g^{\mu \nu}=0$ and $g^{0 i}=0$. We note that for such a coordinate system time $(\eta)$ translations are an isometry, so that $\frac{\partial}{\partial \eta}=K^{\mu}=(1,0, \ldots, 0)$ is a Killing vector [15].

We shall find the $(\vec{\xi}, \eta)$ coordinate transformation by comparing the expression for $K$ in the $(\vec{x}, t)$ coordinate system with $K$ in the $(\vec{\xi}, \eta)$ coordinate system. 
In the $(\vec{x}, t)$ coordinate system, we know that $K$ must be a linear combination of the ten Killing vectors of Minkowski space:

$$
K=\sum_{\alpha=0}^{d} \tau^{\alpha} T_{(\alpha)}+\sum_{i, j=1}^{d} \tilde{\rho}^{i j} R_{(i j)}+\sum_{i=1}^{d} \beta^{i} \vec{B}_{(i)} .
$$

where $\tilde{\rho}_{i j}$ is an antisymmetric matrix, $T_{(\alpha)}$ are the Killing vectors corresponding to translations, $R_{(i j)}$ are Killing vectors corresponding to rotations in the $x_{i}-x_{j}$ plane, and $B_{i}$ to rotations in the $\left(t-x_{i}\right)$ plane (boosts in the $x_{i}$ direction). In order for $K$ to be time-like, we need that either $\tau_{0} \neq 0$ or that $\beta_{i} \neq 0$ for some $i$ (or both). In the latter case, this allows for several simplifications of equation (15).

\section{A. The $\vec{\beta} \neq 0$ case}

We may simplify the boost term, $\sum_{i=1}^{d} \beta^{i} \vec{B}_{(i)}$ in equation (15), by rotating the $\vec{x}$ coordinates [16]: a linear combination of two boost generators is a boost generator in another direction, so by choosing this direction to be, say, the 1 direction, we may bring (by a spatial rotation in the cartesian $(\vec{x}, t)$ coordinate system) the expression $\sum_{i=1}^{d} \beta^{i} \vec{B}_{(i)}$ to the form $\beta \vec{B}_{(1)}$.

The translation in the direction of the boost may be eliminated by a shift in the origin of the time coordinate - that is, the axis of rotation for the boost will be moved. Explicitly $\beta B_{(x)}+a T_{(x)}=\beta\left(t+\frac{a}{\beta}\right) \partial_{x}-\beta x \partial_{t}$.

Next consider the time-translation. Adding a time translation to a boost results in another boost with a shifted space coordinate, so we may also eliminate the time translation: $\beta B_{(y)}+\tau^{0} T_{(0)}=\beta t \partial_{y}-\left(\beta y+\tau^{0}\right) \partial_{t}$.

Finally, rotations that rotate the direction of the boost may be eliminated by changing the boost axis by an appropriate redefinition of the coordinates. We have thus simplified expression (15) to:

$$
K=\sum_{\alpha=2}^{d} \tau^{\alpha} T_{(\alpha)}+\sum_{i, j=2}^{d} \tilde{\rho}^{i j} R_{(i j)}+\beta \vec{B}_{(1)} .
$$

In order to find the desired coordinate transformation (equation (14)), we compare the expressions we have for $K$. On one hand we know from vector transformation properties that:

$$
\frac{\partial}{\partial \eta}=\frac{\partial t}{\partial \eta} \partial_{t}+\frac{\partial x}{\partial \eta} \partial_{x}+\frac{\partial z_{i}}{\partial \eta} \partial_{z_{i}} .
$$


On the other hand, we have from equation (16)

$$
\partial_{\eta}=\beta x \partial_{t}+\beta t \partial_{x}+\left(\tau_{i}+\sum_{j \neq i>1}^{d} \tilde{\rho}_{j i} z_{j}\right) \partial_{z_{i}}
$$

Where $x$ is the direction along the boost, and $\vec{z}$ are the directions orthogonal to it. We get the following set of equations:

$$
\begin{aligned}
& \frac{\partial t}{\partial \eta}=\beta x \\
& \frac{\partial x}{\partial \eta}=\beta t \\
& \frac{\partial z_{i}}{\partial \eta}=\tau_{i}+\tilde{\rho}_{j i} z_{j}
\end{aligned}
$$

The general solution for the first two equations is

$$
\begin{aligned}
& t=A(\vec{\xi}) \sinh (\beta \eta+\Phi(\vec{\xi})) \\
& x=A(\vec{\xi}) \cosh (\beta \eta+\Phi(\vec{\xi}))
\end{aligned}
$$

up to an exchange of the cosh and sinh functions (which will amount to a redefinition of the time and space coordinates.)

We can write the equation for the orthogonal coordinates as a vector equation:

$$
\dot{\vec{z}}=\vec{\tau}+\tilde{\rho} \vec{z}
$$

This is a non homogeneous linear equation. The most general solution is given by $\vec{z}=$ $O(\eta) \vec{h}(\xi)+\vec{z}_{S}(\eta)$. Where $O_{i j}(\eta) h_{j}(\xi)$ (With $O(\eta)=e^{\tilde{\rho} \eta}$ an orthogonal matrix, and $\vec{h}(\xi)$ an arbitrary vector) is a solution to the homogeneous equation, and $z_{S}(\eta)$ is an arbitrary solution of the non homogeneous equation. We may solve for $z_{S}(\eta)$ explicitly, however, the expression is not necessary for the rest of our calculations, and it is not very illuminating (note that $\tilde{\rho}$ is not necessarily invertible.) We emphasize that $\vec{z}_{S}(\eta)$ is independent of $\vec{\xi}$, since one can always treat $\vec{z}$ as a function of $\eta$ only when looking for a special solution of (20).

To simplify matters further, we note that one may arbitrarily reparametrize the $\vec{\xi}$ coordinates, without changing the stationary properties of the metric. We shall use this freedom 
to define new spatial coordinates: $\zeta_{1}=A(\vec{\xi})$, and $\zeta_{i}=h_{i}(\vec{\xi})$,

$$
\begin{aligned}
t & =\zeta_{1} \sinh (\beta \eta+\Phi(\zeta)) \\
x & =\zeta_{1} \cosh (\beta \eta+\Phi(\zeta)) \\
z_{i} & =O_{i j} \zeta_{j}+z_{S i}(\eta) .
\end{aligned}
$$

Now we impose the boundary conditions specified by eq.(15) (in Euclidian space). Starting with the first boundary condition (equation (5a)), we need that the hypersurface $\left\{\left(\vec{x}, 0^{+}\right) \mid \vec{x} \in V\right\}$ coincide with the hypersurface $\left\{(\vec{\zeta}, 0) \mid \vec{\zeta} \in I_{\zeta}\right\}$. To verify this we rotate the time coordinates in equations (21) to the imaginary axis, and check that the hypersurfaces are equal. We get:

$$
\begin{aligned}
& x=\zeta_{1} \\
& \vec{z}=\vec{\zeta}_{\perp}+\vec{z}_{S}(0) .
\end{aligned}
$$

Note that $\vec{z}_{S}(0)$ is a constant vector.

Next, we check the equality of the hypersurfaces at $t=0^{-}$(equation $(5 \mathrm{~b})$ ). We get that in this case:

$$
\begin{aligned}
& 0=\zeta_{1} \sin \left(\beta \beta_{0}\right) \\
& x=\zeta_{1} \cos \left(\beta \beta_{0}\right) \\
& \vec{z}=O\left(\imath \beta_{0}\right) \vec{\zeta}_{\perp}+\vec{z}_{S}\left(\imath \beta_{0}\right) .
\end{aligned}
$$

This implies that $\beta=\frac{2 \pi}{\beta_{0}}$, and that

$$
O\left(\imath \beta_{0}\right) \vec{\zeta}_{\perp}+\vec{z}_{S}\left(\imath \beta_{0}\right)=\vec{\zeta}_{\perp}+\vec{z}_{S}(0)
$$

In order for this equality to hold one needs that the $\zeta$ dependent terms be equal, and that the constant terms be equal.

Starting with the $\zeta$ dependent terms we have: $O\left(\imath \beta_{0}\right)_{i j} \zeta_{j}=\zeta_{i}$. Therefore, $O\left(\imath \beta_{0}\right)=\delta_{i j}$, implying that $\tilde{\rho}=0$. In this case $\vec{z}_{S}(\eta)$ simplifies to $\vec{z}_{S}(\eta)=\vec{\tau} \eta$, which according to equation (24) gives us $\vec{\tau}=0$.

We find that the desired coordinate transformation must satisfy:

$$
\begin{aligned}
& t=\zeta_{1} \sinh (\beta \eta) \\
& x=\zeta_{1} \cosh (\beta \eta) \\
& \vec{z}=\vec{\zeta}_{\perp} .
\end{aligned}
$$


This is a polar coordinate transformation in the $(\vec{x}, t)$ plane. In order that it be onto, we need that $V$ (assumed to be connected) is half of space.

\section{B. The $\tau_{0} \neq 0$ case}

Here, we do not need to simplify equation (15), as all the $\beta_{i}$ vanish. The equivalent of equations (18) are

$$
\begin{aligned}
& \frac{\partial t}{\partial \eta}=\tau_{0} \\
& \frac{\partial x}{\partial \eta}=\tau_{i}+\sum_{j, i}^{d-1} \tilde{\rho}_{j i} x_{j}
\end{aligned}
$$

Whose solution is:

$$
\begin{aligned}
t & =\tau_{0} \eta+A(\vec{\zeta}) \\
z_{i} & =O_{i j} \zeta_{j}+z_{S_{i}}(\eta)
\end{aligned}
$$

Imposing the first boundary condition (equation (5a ) ), we get that $A=0$, and that $\vec{z}=\vec{\zeta}+\vec{z}_{S}(0)$. For the second condition to hold (equation (5b) ), we need that $\tau_{0}=0$ which is ruled out since then $K$ is not timelike. We note that there is a further possibility: if we identify the limits $t \rightarrow \infty$ and $t \rightarrow-\infty$ (meaning, we compactify the time coordinate on an infinitely large circle), then in the limit where $\beta_{0}$ is of the order of the circumference of the time direction $(\beta \rightarrow \infty)$, we may have $\lim _{\eta \rightarrow \beta_{0}} \tau_{0} \eta=0^{-}$. In this case, we also get $\tilde{\rho}=0$ and $\vec{\tau}=0$ (following arguments similar to the $\vec{\beta} \neq 0$ case), so that:

$$
\begin{aligned}
t & =\tau_{0} \eta \\
z_{i} & =\zeta_{j},
\end{aligned}
$$

which may be reduced to the identity transformation, giving back Minkowski space. In order for the transformation to be onto, we obviously need that $V$ be all of space.

What we have shown then, is that if we wish to identify $H_{\text {eff }}$ as the Hamiltonian of a free field in a static curved background, then $V$ must be half of space or all of space, corresponding to a Rindler coordinate transformation, or no transformation at all. 


\section{IMPLICATIONS AND SUMMARY}

We have discussed two types of observers. One makes measurements which are restricted to a certain volume $V$ of Minkowski space. It has been shown elsewhere [8, 12], that this observer's measurements will have fluctuations which scale linearly with the surface area. A second observer has no access to anything external to $V$. This observer sees a state described by a density matrix $\rho_{i n}$. The averages she will measure are equal to expectation values of measurements of the first observer who chooses to restrict his measurements to $V$. This was shown in section II.

Since fluctuations of measurements for both observers will, generally, scale linearly with the surface area, we have looked for the 'effective Hamiltonian' of the second observer. It was shown that if the volume is half of space, there exists a 'natural' Hamiltonian for such an observer: the Rindler Hamiltonian. Otherwise, we have not found an interpretation of $H_{\text {eff }}$. We did show that it can not be the Hamiltonian of a free field in a static curved, or geometrically non-trivial, background.

In the case where the volume is half of space, the Rindler observer and the observer restricting his measurements to half of space are equivalent. This enables us to calculate statistical averages for the Rindler observer by calculating quantum expectation values of the Minkowski observer. (This is related to the fact the left Rindler wedge is the thermofield double of the right Rindler wedge.) We have used this correspondence to explicitly calculate the heat capacity of Rindler space radiation, by calculating the quantum fluctuations of the Rindler Hamiltonian in the Minkowski vacuum.

We should emphasize, that even though we have no knowledge of its attributes, the system described by $\rho_{i n}$ exhibits area scaling of fluctuations. Since non-extensivity of fluctuations exists for any volume, area scaling for a volume which is half of space is only a special case of area scaling of thermodynamic quantities. In this sense, one may argue that the area scaling of Rindler space thermodynamics is a special case of a more general phenomenon.

Finally, we have shown in [8, 13] that the area scaling of fluctuations can lead to a bulk-boundary correspondence. With our proposed relation between Rindler space and an observer making measurements in half of Minkowski space, this may lead to an interesting analogy with a presumed holographic reduction of Rindler space. 


\section{ACKNOWLEDGMENTS}

Research supported in part by the Israel Science Foundation under grant no. 174/002 and by the NSF under grant no.PHY-99-07949. A. Y. is partially supported by the Kreitman foundation. R. B. thanks the KITP, UC at Santa Barbara, where this work has been completed. We would like to thank S. de Alwis, and Y. Lederer for helpful discussions.

\section{APPENDIX A: CALCULATION OF ENERGY FLUCTUATIONS IN RINDLER SPACE.}

We briefly go over the calculations in [12], which give the energy fluctuations of the Rindler space Hamiltonian in half of Minkowski space.

We first recall the calculation of energy fluctuations in a half of Minkowski space. We define the energy operator for a general sub-volume $V$ as

$$
E^{V}=\int_{V} \mathcal{H}(\vec{x}) d^{d} x
$$

where $\mathcal{H}(\vec{x})$ is the $T^{00}$ component of the energy momentum tensor, and we are working in the Schroedinger picture. For a free field theory, we have

$$
\begin{array}{r}
: E^{V}:=\frac{1}{4} \frac{1}{(2 \pi)^{2 d}} \int\left(\left(\frac{-\vec{p} \cdot \vec{q}}{\sqrt{\omega_{p} \omega_{q}}}-\sqrt{\omega_{p} \omega_{q}}\right)\left(a_{\vec{p}} a_{\vec{q}}+a_{-\vec{p}} a_{-\vec{q}}\right)+2\left(\frac{-\vec{p} \cdot \vec{q}}{\sqrt{\omega_{p} \omega_{q}}}+\sqrt{\omega_{p} \omega_{q}}\right) a_{-\vec{p}} a_{\vec{q}}\right) \\
\times e^{\imath(\vec{p}+\vec{q}) \cdot \vec{x}} d^{d} p d^{d} q d^{d} x .
\end{array}
$$

We see that $\left\langle: E_{V}:\right\rangle=0$, and proceed to calculate $\left\langle: E_{V}:{ }^{2}\right\rangle$. We get:

$$
\left\langle: E^{V}:^{2}\right\rangle=\frac{1}{8} \frac{1}{(2 \pi)^{2 d}} \iiint_{V} \int_{V}\left(\frac{-\vec{p} \cdot \vec{q}}{\sqrt{\omega_{p} \omega_{q}}}-\sqrt{\omega_{p} \omega_{q}}\right)^{2} e^{\imath(\vec{p}+\vec{q}) \cdot(\vec{x}-\vec{y})} d^{d} p d^{d} q d^{d} x d^{d} y .
$$

Since after integration over the momenta we get an integration over the variable $|\vec{x}-\vec{y}|$, we may rewrite this integral as $\int_{0}^{\infty} F(\xi) D(\xi) d \xi$, where for a free field theory,

$$
\begin{aligned}
F(x) & =\frac{1}{8} \frac{1}{(2 \pi)^{2 d}} \iint\left(\frac{-\vec{p} \cdot \vec{q}}{\sqrt{\omega_{p} \omega_{q}}}-\sqrt{\omega_{p} \omega_{q}}\right)^{2} e^{\imath(\vec{p}+\vec{q}) \cdot(\vec{x}-\vec{y})} d^{d} p d^{d} q \\
& =\frac{1}{8} \frac{1}{(2 \pi)^{2 d}} \int\left(p q+2 \vec{p} \cdot \vec{q}+\frac{(\vec{p} \cdot \vec{q})^{2}}{p q}\right) e^{-\imath(\vec{p}+\vec{q}) \cdot \vec{x}} d^{d} p d^{d} q
\end{aligned}
$$

and

$$
D(\xi)=\int_{V} \int_{V} \delta^{(d)}(\xi-|\vec{x}-\vec{y}|) d^{d} x d^{d} y .
$$


We first consider $F(x)$. We switch to a coordinate system where:

$$
\vec{x}=\left(\begin{array}{c}
x \\
0 \\
0 \\
\vdots \\
0
\end{array}\right) ; \vec{q}=\left(\begin{array}{c}
q_{x} \\
q_{\perp} \\
0 \\
\vdots \\
0
\end{array}\right) ; \vec{p}=\left(\begin{array}{c}
p_{x} \\
p_{\perp} \cos \theta_{p} \\
p_{\perp \perp} \\
\vdots \\
0
\end{array}\right) .
$$

In this form, we may do all angular integrations:

$$
\begin{aligned}
F(x)=\frac{1}{8} \frac{1}{(2 \pi)^{2 d}}\left(\frac{\pi^{\frac{d}{2}}}{\Gamma\left(\frac{d}{2}+1\right)}(d-1)\right)^{2} & \\
\times \int\left(p q+2 p_{x} q_{x}+\frac{p_{x}^{2} q_{x}^{2}}{p q}+\right. & \left.\frac{p_{\perp}^{2} q_{\perp}^{2}}{p q} \frac{1}{d-1}\right) \\
& \times e^{-\imath\left(p_{x}+q_{x}\right) x} p_{\perp}^{d-2} q_{\perp}^{d-2} d p_{\perp} d q_{\perp} d p_{x} d q_{x} .
\end{aligned}
$$

Switching to polar coordinates in the remaining two dimensional system:

$$
\begin{aligned}
& p_{\perp}=p \sin \theta \\
& p_{x}=p \cos \theta,
\end{aligned}
$$

and observing that integrations over the $p$ and $q$ variables are independent, we can now evaluate the integral with an exponential high momentum cutoff:

$$
\begin{aligned}
F(x) & =\frac{(d+1) \Gamma\left(\frac{d+1}{2}\right) \Lambda^{2(d+1)}}{8 \pi^{d+1}\left(1+(\Lambda x)^{2}\right)^{d+3}}\left(d-2(d+2)(\Lambda x)+d(\Lambda x)^{4}\right. \\
& =\frac{(d+1) \Gamma\left(\frac{d+1}{2}\right) \Lambda^{2(d+1)}}{8 \pi^{d+1}} \nabla_{\Lambda x}^{2} \frac{\left.(\Lambda x)^{2}-1\right)}{2(d+2)\left(1+(\Lambda x)^{2}\right)^{d+1}} .
\end{aligned}
$$

Next we consider the geometric term for the specific case that $V$ is half of Minkowski space.

$$
D(\xi)=\int_{-\infty}^{\infty} \ldots \int_{-\infty}^{\infty} \int_{0}^{\infty} \int_{0}^{\infty} \delta^{(d)}\left(\xi-\left|\vec{x}_{1}-\vec{x}_{2}\right|\right) d^{d} x_{1} d^{d} x_{2} .
$$

Switching to $\vec{r}_{ \pm}=\vec{x}_{1} \pm \vec{x}_{2}$ coordinates, we may integrate over the transverse $\vec{r}_{+}$directions, yielding the transverse volume $V_{\perp}$ (here transverse means the directions transverse to the $z$ coordinate.) Therefore:

$$
D(\xi)=\frac{1}{2} V_{\perp} \int_{0}^{\infty} \int_{-z_{+}}^{z_{+}}\left[\int_{-\infty}^{\infty} \ldots \int_{-\infty}^{\infty} \delta^{(d)}\left(\xi-r_{-}\right) d^{d-1} r_{-\perp}\right] d z_{-} d z_{+} .
$$


Putting this back in equation (A1) and substituting $r_{-} \rightarrow \Lambda r_{-}$, we have

$$
\left\langle: E^{V^{2}}:\right\rangle=\frac{1}{2} V_{\perp} \Lambda^{d-1} \int_{0}^{\infty} \int_{-z_{+}}^{z_{+}}\left[\int_{-\infty}^{\infty} \ldots \int_{-\infty}^{\infty} F_{d}\left(\frac{r_{-}}{\Lambda}\right) d^{d-1} r_{-\perp}\right] d z_{-} d z_{+} .
$$

Working in a d-dimensional 'cylindrical' coordinate system $\left(d \vec{x}^{2}=d \rho^{2}+d z_{-}^{2}+\rho^{d-2} d \Omega^{2}\right)$, we may easily do all angular integrations. So that

$$
\begin{aligned}
\left\langle: H^{V^{2}}:\right\rangle \propto \int_{0}^{\infty} \int_{-z_{+}}^{z_{+}} \int_{0}^{\infty}\left[\frac { \partial } { \partial \rho } \left(\rho^{d-2} \frac{\partial}{\partial \rho}(\right.\right. & \left.\left.\frac{\rho^{2}+z_{-}^{2}-1}{2(d+2)\left(1+\rho^{2}+z_{-}^{2}\right)^{d+1}}\right)\right) \\
& \left.+\rho^{d-2} \frac{\partial^{2}}{\partial z_{-}^{2}}\left(\frac{\rho^{2}+z_{-}^{2}-1}{2(d+2)\left(1+\rho^{2}+z_{-}^{2}\right)^{d+1}}\right)\right] d \rho d z_{-} d z_{+},
\end{aligned}
$$

where numerical coefficients have been omitted for clarity. The first term vanishes upon integration, and the second term may be calculated explicitly. The final result is:

$$
\left\langle: E^{V^{2}}:\right\rangle=V_{\perp} \Lambda^{d+1} \frac{(d+1) \Gamma^{2}\left(\frac{d+1}{2}\right)}{2^{d+5} \pi^{1+\frac{d}{2}} \Gamma\left(2+\frac{d}{2}\right)} .
$$

Hence energy fluctuations are proportional to the surface area $V_{\perp}$.

As the Rindler Hamiltonian is in essence the 'boost' generator, we proceed to calculate fluctuations of the boost generator in the ' $z$ ' direction, $B_{(z)}^{V}$. Since [14]

$$
B_{(z)}^{V}=\int_{V} z \mathcal{H} d^{d} x
$$

Using the same notation as in the previous calculation, we get that $\left\langle: B_{(z)}^{V}:\right\rangle=0$, and

$$
\begin{aligned}
\left\langle:\left(B_{(z)}^{V}\right)^{2}:\right\rangle & =\int z_{1} z_{2} F_{d}\left(r_{-}\right) d^{d} x_{1} d^{d} x_{2} \\
& =\frac{1}{2} V_{\perp} \int_{0}^{\infty} \int_{-z_{+}}^{z_{+}}\left[\int_{-\infty}^{\infty} \ldots \int_{-\infty}^{\infty} \frac{1}{4}\left(z_{+}^{2}-z_{-}^{2}\right) F_{d}\left(r_{-}\right) d^{d-1} r_{-\perp}\right] d z_{-} d z_{+} .
\end{aligned}
$$

This integral may now be evaluated as before, giving

$$
\left\langle:\left(B_{(z)}^{V}\right)^{2}:\right\rangle=\frac{1}{\Lambda^{2}} \frac{1}{d-1}\left\langle: E^{V}:^{2}\right\rangle .
$$

Here, again, the fluctuations are proportional to the surface area. The Rindler Hamiltonian is given by $H_{R}=a B_{(z)}^{V}$, so that $\left\langle: H_{R}:^{2}\right\rangle=a^{2}\left\langle:\left(B_{(z)}^{V}\right)^{2}:\right\rangle$.

[1] M. Srednicki, Phys. Rev. Lett. 71, 666 (1993), hep-th/9303048. 
[2] L. Bombelli, R. K. Koul, J. H. Lee and R. D. Sorkin, Phys. Rev. D 34, 373 (1986).

[3] C. Callan and F. Wilczek, Phys. Lett. B333, 55 (1994), hep-th/9401072

[4] S. P. de Alwis and N. Ohta, (1994), hep-th/9412027.

[5] S. P. de Alwis and N. Ohta, Phys. Rev. D52, 3529 (1995), hep-th/9504033.

[6] C. Holzhey, F. Larsen, and F. Wilczek, Nucl. Phys. B424, 443 (1994), hep-th/9403108

[7] L. Susskind and J. Uglum, Phys. Rev. D50, 2700 (1994), hep-th/9401070

[8] R. Brustein and A. Yarom, (2003), hep-th/0302186.

[9] R. P. Feynman, Statistical Mechanics: A Set of Lectures (Perseues Publishing, 1998).

[10] D. Kabat and M. J. Strassler, Phys. Lett. B329, 46 (1994), hep-th/9401125

[11] R. Emparan, Phys. Rev. D 51, 5716 (1995), hep-th/9407064

[12] R. Brustein and A. Yarom, to appear.

[13] R. Brustein, D. Oaknin, and A. Yarom, "Implications of area scaling of quantum fluctuations," hep-th/0310091.

[14] S. Weinberg, The Quantum Theory of Fields V 1 (Cambridge University Press, Cambridge, U.K., 2001).

[15] Actually, this statement is correct even if $g^{0 i} \neq 0$. However, we will see that once we impose the boundary conditions, we shall get $g^{0 i}=0$ automatically.

[16] The fact that rotations in the $(\vec{x}, t)$ coordinate system do not change the stationary character of the metric in the $(\xi, \eta)$ coordinate system follows from the fact that rotations (and similarly translations and boosts) are Killing transformations and leave the metric invariant. 